\title{
Does mass spectrometric breath analysis detect Pseudomonas aeruginosa in cystic fibrosis?
}

\begin{abstract}
To the Editor:
Pseudomonas aeruginosa associated with increased morbidity and mortality in cystic fibrosis (CF) can be eradicated if detected early; however, prompt detection is problematic in children and non-expectorating adults as oropharyngeal cultures lack sensitivity [1]. We previously reported that $20 \%$ of children with negative oropharyngeal cultures isolated $P$. aeruginosa on bronchoalveolar lavage [2], but this invasive technique cannot be performed frequently. Hence, there is an urgent need for a noninvasive screening test for lower airways infection in CF; therefore, we propose that exhaled volatile organic compounds (VOC) may be useful biomarkers.
\end{abstract}

Over 250 VOC have been detected in exhaled breath using mass spectrometry. P. aeruginosa has a characteristic odour related to VOC, such as 2-aminoacetophenone (2-AA) [3]; 2-AA, hydrogen cyanide $(\mathrm{HCN})$ and other compounds have been detected in the exhaled breath of $P$. aeruginosa-infected CF patients [4]. Selected ion-flow tube mass spectrometry (SIFT-MS) allows simultaneous quantification of multiple VOC without costly and time consuming pre-concentration steps [5]. Herein, we report the most comprehensive analysis to date of exhaled VOC using SIFT-MS in CF patients. We hypothesised that differences in VOC profile can distinguish CF patients with and without chronic P. aeruginosa infection.

Following informed consent, adults with CF were recruited from two groups: those with chronic $P$. aeruginosa infection and those without $P$. aeruginosa infection based on the Leeds criteria [6].

Breath sampling and analytical procedures were conducted using a validated method [7]. Following $\geqslant 4 \mathrm{~h}$ of fasting, subjects provided single exhalations from total lung capacity into double-thickness $(2 \times 25 \mu \mathrm{m})$ single-use, 2 L Nalophan bags (BOC Ltd, Guildford, UK) pre-flushed with synthetic air. Subjects inhaled fully through the nose (as close to total lung capacity as possible) and exhaled slowly from the mouth directly into the sampling bag. All samples were analysed within $2 \mathrm{~h}$ of collection by a Profile 3 SIFT-MS instrument (Instrument Science, Crewe, UK) using full scan and multiple ion monitoring modes as previously described (figure 1a) [7].

Statistical analyses were performed using SPSS Statistics 21.0 (IBM, Armonk, NY, USA) and Prism 6.0 (GraphPad, La Jolla, CA, USA). VOC were incorporated into a binary logistic regression model to discriminate between CF patients with and without P. aeruginosa infection. Accuracy of the diagnostic models was assessed using the area under the curve (AUC) of the receiver operating characteristics (ROC) curves. The null hypothesis was rejected at $\mathrm{p}<0.05$.

In the CF patients without $P$. aeruginosa infection $(n=29)$, the median (interquartile range (IQR)) number of culture samples negative for $P$. aeruginosa growth over the preceding 12 months was 8 (5-11). For the CF patients with $P$. aeruginosa infection $(n=44)$ the median $(I Q R)$ number of cultures samples was 9 (717). Categorised by most recent culture, $14(31.8 \%)$ had non-mucoid strains, six (13.6\%) had mucoid strains and 24 (54.5\%) had both phenotypes. CF patients with and without $P$. aeruginosa infection were matched for age, sex, body mass index, CF transmembrane conductance regulator genotype, pancreatic insufficiency, IgE, IgE specific to Aspergillus fumigatus, rates of CF-related diabetes and liver disease, and percentage of water levels in exhaled breath. Forced expiratory volume in $1 \mathrm{~s}$ (FEV1) was lower in CF patients with $P$. aeruginosa infection (median (IQR) 45 (28.3-61.8) versus 57 (46.0-74.5), $\mathrm{p}<0.05$ ).

Linear regression analysis showed that any correlations of VOC with FEV1 were weak (e.g. propanol: $r^{2}=0.066$; dimethyl disulfide (DMDS): $r^{2}=0.097$ ), and thus contributed little to any $P$. aeruginosa-related observations.

Of the 12 VOC, significant differences between the CF patients with and without $P$. aeruginosa infection were seen in three VOC (figure $1 b)$. DMDS $(p<0.05)$ and 2 -AA were higher $(p<0.01)$ in the CF patients with $P$. aeruginosa infection and butanol was lower $(\mathrm{p}<0.01)$. ROC constructed using binary regression of these three VOC resulted in an AUC of 0.774 (95\% CI $0.667-0.882)$ with a sensitivity and specificity of 0.828 and 0.649 , respectively. However, using all 12 VOC improved the discrimination of CF patients with and without $P$. aeruginosa infection with an AUC of 0.842 (95\% CI $0.753-0.930)$, sensitivity of 0.828 and specificity of 0.705 (figure $1 \mathrm{c}$ and $\mathrm{d}$ ). 

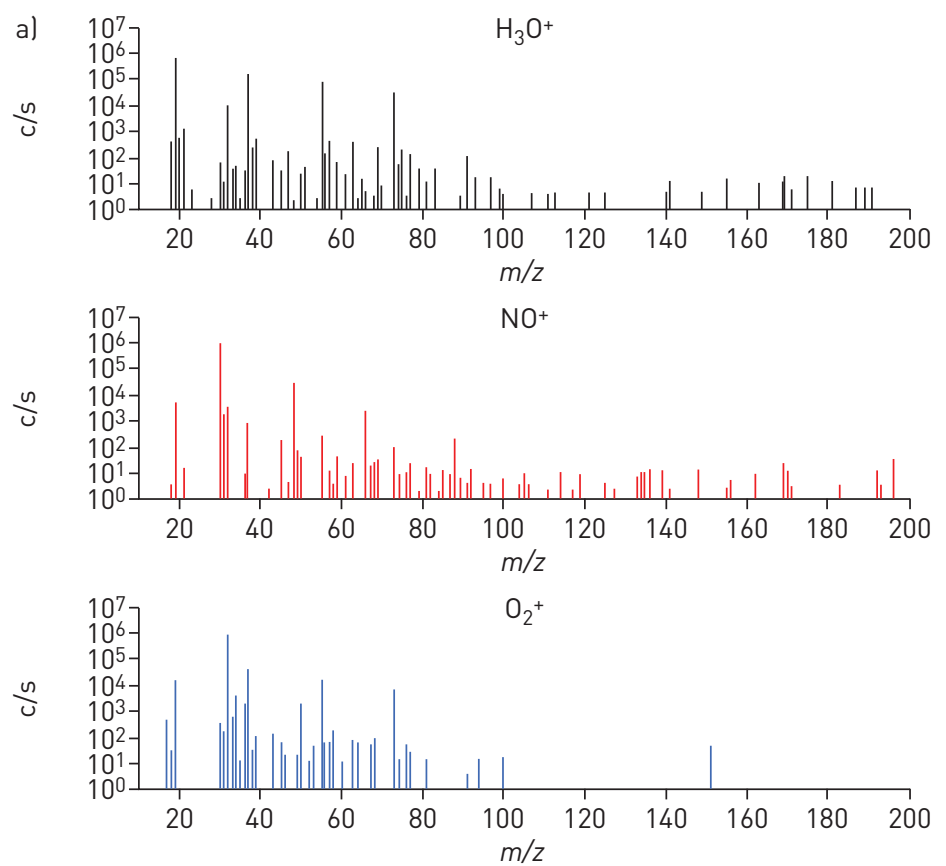
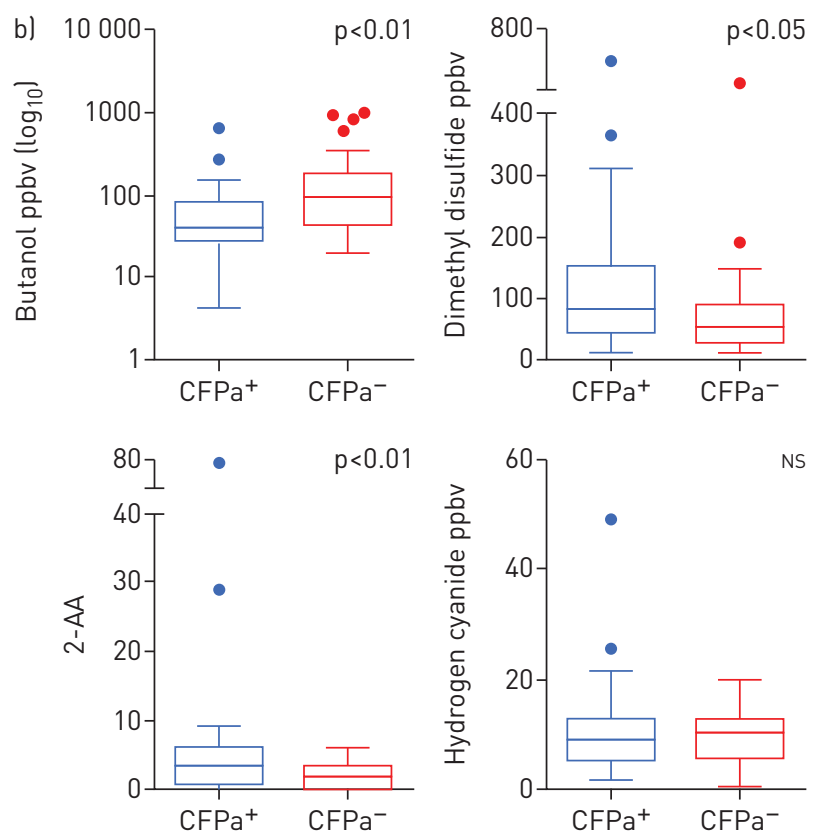
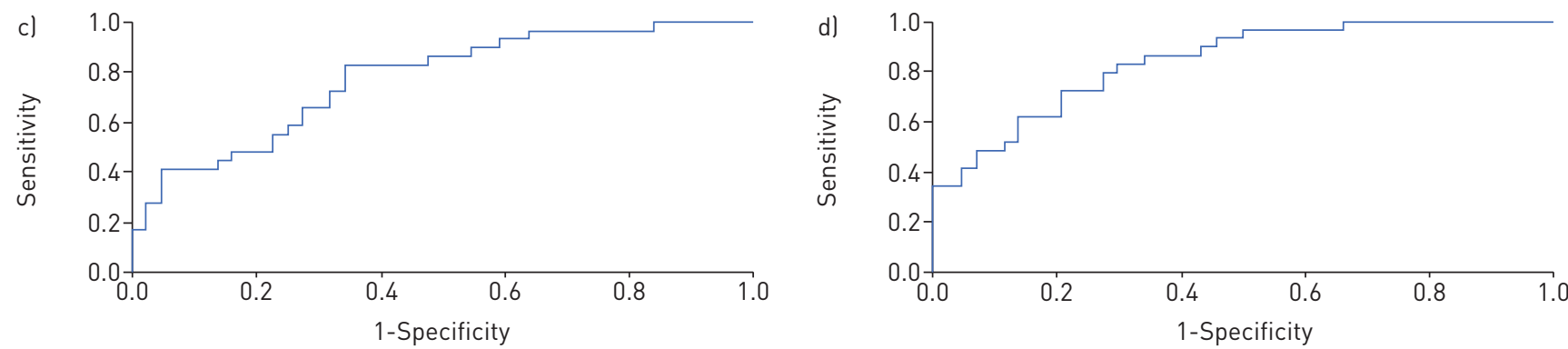

Figure 1 a) Full scan spectrum of exhaled breath from cystic fibrosis (CF) patients analysed using the $\mathrm{H}_{3} \mathrm{O}^{+}$precursor ion, $\mathrm{NO}^{+}$precursor ion and $\mathrm{O}_{2}^{+}$ precursor ion. The individual characteristic product ions and their hydrates were assigned to the analysed molecules. In $\mathrm{H}_{3} \mathrm{O}^{+}, \mathrm{m} / z \mathrm{z}, 37,55$ and 73 are $\mathrm{H}_{3} \mathrm{O}+\left(\mathrm{H}_{2} \mathrm{O}\right)_{0,1,2,3} ; \mathrm{m} / \mathrm{z} 33$ and 51 are methanol; $\mathrm{m} / \mathrm{z} 83$ is ethanol; $\mathrm{m} / \mathrm{z} 83$ is propanol and its monohydrate product ion; $\mathrm{m} / \mathrm{z} 57$ and 75 are butanol and its monohydrate product ion; $\mathrm{m} / \mathrm{z} 71$ and 89 are pentanol and its monohydrate product ion; $\mathrm{m} / \mathrm{z} 35$ is hydrogen sulfide; $\mathrm{m} / \mathrm{z} 28$ is hydrogen cyanide; $\mathrm{m} / z 97$ is dimethyl disulfide. In $\mathrm{NO}^{+}, \mathrm{m} / z 19,37,55$ and 73 are $\mathrm{H}_{3} \mathrm{O}+\left(\mathrm{H}_{2} \mathrm{O}\right)_{0,1,2,3} ; \mathrm{m} / z$ 30, 48,66 and 84 are $\mathrm{NO}+\left(\mathrm{H}_{2} \mathrm{O}\right)_{0,1,2,3 ;} \mathrm{m} / \mathrm{z} 68$ is isoprene; $\mathrm{m} / z 88$ is acetone; $\mathrm{m} / \mathrm{z} 135$ is 2-AA. In $\mathrm{O}_{2}^{+}, \mathrm{m} / \mathrm{z} 19,37,55$ and 73 are $\mathrm{H}_{3} \mathrm{O}+\left(\mathrm{H}_{2} \mathrm{O}\right)_{0,1,2,3} ; \mathrm{m} / \mathrm{z} 32,50$ and 68 are $\mathrm{O}_{2}+\left(\mathrm{H}_{2} \mathrm{O}\right)_{0,1,2} ; \mathrm{m} / \mathrm{z} 17$ and 35 are ammonia. c/s: counts per second. b) Box and whisker plots (Tukey) demonstrating significant differences in butanol, dimethyl disulfide and 2-aminoacetophenone (2-AA) in the exhaled breath of CF patients with and without Pseudomonas aeruginosa (CFPa+ and CFPa-, respectively). No difference is demonstrated in mouth exhaled hydrogen cyanide between the groups. Ns: nonsignificant. C) Receiver operating characteristic (ROC) curve for CFPa+ versus CFPa- patients with only three significantly different volatile organic compounds (butanol, dimethyl disulfide and 2-AA) used in the binary regression model. d) ROC curve for CFPa+ versus CFPa- patients with all 12 volatile organic compounds used in the binary regression model.

The feasibility of using metabolomics for respiratory diagnosis has previously been demonstrated using nuclear magnetic resonance (NMR) spectroscopy of exhaled breath condensate (EBC) to distinguish between asthmatics and healthy controls [8], subjects with CF and primary ciliary dyskinesia [9], and patients with stable and unstable CF [10]. Here, we demonstrate proof-of-concept that a VOC signature is present in exhaled breath (that, unlike NMR spectroscopy of EBC, has potential for real-time analysis), which differentiates groups of CF patients with and without chronic P. aeruginosa infection. Any individual VOC discriminated relatively poorly, even those in which significant group differences were detected; however, by combining VOC signals, high levels of sensitivity and specificity could be achieved. To our knowledge, it is the most comprehensive breath profiling study in CF patients to date. Whilst encouraging, with greater sensitivity than clinically utilised oropharyngeal cultures [1], this compares unfavourably to, for example, $\left[{ }^{13} \mathrm{C}\right]$ urea breath test for Helicobacter pylori in children (sensitivity $93.8 \%$ and specificity 99.1\%) [11]. Adding other VOC to this "P. aeruginosa fingerprint" might increase discriminatory capacity to a clinically applicable standard, particularly as improved SIFT-MS sensitivity will allow for detection in the ppt range [12]. Combining SIFT-MS with highly sensitive chemical sensor arrays (electronic noses) is another option that could enhance accuracy, although this technology also has limitations that would need to be overcome before clinical use [13]. 
The three markers which demonstrated group differences (2-AA, DMDS and butanol) have all been reported previously as being associated with $P$. aeruginosa infection [14]. However, missing from the group was HCN, which has previously been considered to have biomarker potential. Raised HCN levels have been previously reported in $P$. aeruginosa-infected sputum using a cyanide sensitive electrode [15], and other groups in vitro above bacterial cultures of $P$. aeruginosa with mass spectrometry. In one exhaled breath study, HCN differentiated between CF children with and without P. aeruginosa infection [16]. However, in another study [17] and in our cohort no difference was observed, despite our detected levels being similar to those reported in the literature $[16,17]$. HCN production varies with $P$. aeruginosa phenotype and genotype [16], which may account for the discrepancy in findings. Furthermore, a recent study suggests that the oral cavity is the main contributor to mouth exhaled HCN [18]. As oral bacterial contamination in adult subjects may be greater than in children [16], nasally exhaled HCN is proposed to be a more accurate biomarker in adults [17]. However, given the almost universal presence of sinus disease in CF adults, and common isolation of $P$. aeruginosa from sinus lavage (in up to $73 \%$ of patients [19]), this approach to exhaled breath sampling may not be fully representative of the lower airways; bacterial strains in one study were concordant between upper and lower airways in fewer than $50 \%$ of cases [19].

We were interested to note when combining VOC into a diagnostic prediction model that accuracy was improved when all 12 VOC were incorporated, as opposed to using only the three discriminatory VOC. This suggests that other individual VOC are underpowered because of the large coefficient of variation with some compounds, but still contribute to the sensitivity of the binary regression model. Further prospective study is required to elucidate influence of each individual VOC that did not reach statistical significance.

This cross-sectional study has some limitations. Although subjects fasted to reduce the potential influence of oral contamination on VOC concentration, it was not possible to stratify patients by concurrent medications (other than inhaled antibiotics) due to the relatively small sample size and the vast number of drug/dose combinations. It is possible that some medications, particularly if inhaled, might influence VOC profile; this needs to be considered during future studies. Finally, the cohort of CF patients with $P$. aeruginosa infection in this initial proof-of-concept study comprised adult patients capable of expectorating sputum and chronically infected with $P$. aeruginosa. For a novel diagnostic screening test to be valuable, any signal would need to be sufficiently sensitive to discriminate between noninfected patients and those with early infection, probably with low bacterial numbers.

In summary, we confirm that by combining a series of measured VOC, CF patients with and without chronic $P$. aeruginosa infection can be differentiated on a group basis. The test is not sufficiently sensitive to be used on an individual case basis and significant improvements are probably needed before this can be applied to the population most in need, young patients with early infection.

0 @ERSpublications

Detecting $P$. aeruginosa infection is problematic; breath analysis shows promise but requires optimisation http://ow.ly/WE1H2

Rishi Pabary ${ }^{1,2}$, Juzheng Huang ${ }^{3}$, Sacheen Kumar ${ }^{3}$, Eric W.F.W. Alton ${ }^{1}$, Andrew Bush ${ }^{1,2}$, George B. Hanna ${ }^{3}$ and Jane C. Davies ${ }^{1,2}$

${ }^{1}$ National Heart and Lung Institute, Imperial College, London, UK. ${ }^{2}$ Dept of Paediatric Respiratory Medicine, Royal Brompton Hospital, London, UK. ${ }^{3}$ Dept of Surgery and Cancer, St Mary's Hospital, London, UK.

Correspondence: Rishi Pabary, National Heart and Lung Institute, Imperial College, Emmanuel Kaye Building, 1b Manresa Road, London, SW3 6LR, UK. E-mail: r.pabary11@imperial.ac.uk

Received: April 192015 | Accepted after revision: Dec 062015 | First published online: Feb 042016

Support statement: This project was funded by the NIHR Respiratory Disease Biomedical Research Unit at the Royal Brompton and Harefield NHS Foundation Trust and Imperial College London, UK. The views expressed in this publication are those of the authors(s) and not necessarily those of the NHS, The National Institute for Health Research or the Department of Health.

Conflict of interest: None declared.

\section{References}

1 Rosenfeld M, Emerson J, Accurso F, et al. Diagnostic accuracy of oropharyngeal cultures in infants and young children with cystic fibrosis. Pediatr Pulmonol 1999; 28: 321-328.

2 Hilliard TN, Sukhani S, Francis J, et al. Bronchoscopy following diagnosis with cystic fibrosis. Arch Dis Child 2007; 92: 898-899.

3 Cox CD, Parker J. Use of 2-aminoacetophenone production in identification of Pseudomonas aeruginosa. J Clin Microbiol 1979; 9: 479-484.

4 Sethi S, Nanda R, Chakraborty T. Clinical application of volatile organic compound analysis for detecting infectious diseases. Clin Microbiol Rev 2013; 26: 462-475. 
5 Smith D, Spanel P. Selected ion flow tube mass spectrometry (SIFT-MS) for on-line trace gas analysis. Mass Spectrom Rev 2005; 24: 661-700.

6 Lee TW, Brownlee KG, Conway SP, et al. Evaluation of a new definition for chronic Pseudomonas aeruginosa infection in cystic fibrosis patients. J Cyst Fibros 2003; 2: 29-34.

7 Kumar S, Huang J, Abbassi-Ghadi N, et al. Selected ion flow tube mass spectrometry analysis of exhaled breath for volatile organic compound profiling of esophago-gastric cancer. Anal Chem 2013; 85: 6121-6128.

8 Sinha A, Krishnan V, Sethi T, et al. Metabolomic signatures in nuclear magnetic resonance spectra of exhaled breath condensate identify asthma. Eur Respir J 2012; 39: 500-502.

9 Montuschi P, Paris D, Montella S, et al. Nuclear magnetic resonance-based metabolomics discriminates primary ciliary dyskinesia from cystic fibrosis. Am J Respir Crit Care Med 2014; 190: 229-233.

10 Montuschi P, Paris D, Melck D, et al. NMR spectroscopy metabolomic profiling of exhaled breath condensate in patients with stable and unstable cystic fibrosis. Thorax 2012; 67: 222-228.

11 de Carvalho Costa Cardinali L, Rocha GA, Rocha AM, et al. Evaluation of $\left[{ }^{13} \mathrm{C}\right]$ urea breath test and Helicobacter pylori stool antigen test for diagnosis of $\mathrm{H}$. pylori infection in children from a developing country. J Clin Microbiol 2003; 41: 3334-3335.

12 Prince BJ, Milligan DB, McEwan MJ. Application of selected ion flow tube mass spectrometry to real-time atmospheric monitoring. Rapid Commun Mass Spectrom 2010; 24: 1763-1769.

13 Montuschi P, Mores N, Trove A, et al. The electronic nose in respiratory medicine. Respiration 2013; 85: 72-84.

14 Goeminne PC, Vandendriessche T, Van Eldere J, et al. Detection of Pseudomonas aeruginosa in sputum headspace through volatile organic compound analysis. Respir Res 2012; 13: 87.

15 Ryall B, Davies JC, Wilson R, et al. Pseudomonas aeruginosa, cyanide accumulation and lung function in CF and non-CF bronchiectasis patients. Eur Respir J 2008; 32: 740-747.

16 Smith D, Spanel P, Gilchrist FJ, et al. Hydrogen cyanide, a volatile biomarker of Pseudomonas aeruginosa infection. J Breath Res 2013; 7: 044001.

17 Dummer J, Storer M, Sturney S, et al. Quantification of hydrogen cyanide (HCN) in breath using selected ion flow tube mass spectrometry - HCN is not a biomarker of Pseudomonas in chronic suppurative lung disease. $J$ Breath Res 2013; 7: 017105.

18 Chen W, Metsala M, Vaittinen O, et al. Hydrogen cyanide in the headspace of oral fluid and in mouth-exhaled breath. J Breath Res 2014; 8: 027108 .

19 Robertson JM, Friedman EM, Rubin BK. Nasal and sinus disease in cystic fibrosis. Paediatr Respir Rev 2008; 9 : 213-219.

\title{
Monoallelic germline ATM mutation and organising pneumonia induced by radiation therapy to the breast
}

\author{
To the Editor:
}

Organising pneumonia is defined by the presence of intra-alveolar buds consisting of inflammatory cells, fibroblasts and myofibroblasts, and loose connective tissue, often associated with bronchiolitis obliterans. Organising pneumonia may be cryptogenic (idiopathic) or associated with a variety of causes including, especially, infection, adverse reaction to drugs, connective tissue diseases [1] and radiation therapy to the breast $[2,3]$.

Ataxia telangiectasia is an autosomal recessive disorder resulting from mutations in the ATM (ataxia telangiectasia mutated) gene located in 11q22.3, with a prevalence of about one in 100000 children. ATM encodes a 350-kDa protein kinase (3056 aminoacyl residues) with catalytic functions similar to phosphatidylinositol 3'-kinases. After DNA damage, especially DNA double-strand breaks, ATM phosphorylates a thousand targets, activating cell cycle checkpoints, DNA repair, and metabolic and senescence pathways. Ataxia telangiectasia is characterised by neurological manifestations, an increased risk of infections, and cancers, especially lymphomas and leukaemias. Ataxia telangiectasia children affected with cancer present with a severe radiosensitivity if radiation is not reduced. Women carrying a monoallelic ATM mutation, for example, the relatives of ataxia telangiectasia-affected children, are at increased risk of breast cancer [4]. The relative risk of breast cancer at age 50 years in ATM carriers has been estimated to be 4.94 (95\% CI 1.90-12.9) among relatives of ataxia telangiectasia patients [5].

A 68-year-old woman underwent tumourectomy for invasive ductal carcinoma of the left breast followed by radiation therapy, completed in September 2008. There were no acute side-effects. The patient did not receive chemotherapy. 2 months later, she developed bilateral pulmonary alveolar opacities on imaging, 